\title{
微处理机在线判定气相色谱法低沸点 碳氢化合物色谱峰
}

\author{
李浩春 戴朝政 徐方宝 卢倵章
}

(中国科学院大连化学物理研究所)

目前最新型的气相色谱仪都有微处理机这个部件. 此微处理机进行色谱峰的定性时, 大 多数只能给出组分的相对保留值, 少数则是要预先在微处理机中存人了组分在规定的固定相 与操作条件相对应的保留值后, 才可以判定色谱峰可能为何物 ${ }^{[1-5]}$. 由于不可能在微处理机 中,直接储存大量组分在任一柱温及不同固定相的保留值 (如保留指数或相对保留值) 供作色 谱峰定性用, 于是我们决意要在色谱保留值理论的引导下,寻求可用最少的参数就能较准确地 计算出组分在不同柱温和不同固定相时保留指数的方法, 并且在微处理机中储存这些参数, 代 替直接储存组分的保留指数供色谱峰定性用. 最近，我们报道了可以用少数参数计算与预测 低沸点异构烷烃、环烷、芳烃保留指数的方法 ${ }^{[6]}$; 本文则是报道我们运用自编的程序,用这些参 数计算出给定柱温及固定相的 $\mathrm{C}_{5}-\mathrm{C}_{3}$ 异构烷烃、环烷、芳烃的保留指数计算值，代替直接储存 组分保留指数的数值,然后对色谱峰进行在线定性的结果.

我们使用的仪器是 Perkin-Elmer 公司 Sigma 1 型气相色谱仪. 它由分析器与控制台所组 成.分析器装有色谱炉、检测器、气流系统、进样系统、温控系统等.控制台有微处理机, 其 RAM 量为 $8 \mathrm{~K}$ 字组, 磁带每面容量为 $15 \mathrm{~K}$ 字组, 字长为 8 组, 可用 BASIC 语言编写程序.

实验时根据样品分析所需的操作条件，按此色谱仪操作规程编排分析方法操作程序,同时 将自编的色谱峰在线定性程序由磁带调人到控制台, 当这些准备工作完成后, 就可进行实验. 实验开始时先从键衁输人使用的固定相的名称及柱温、分析方法号, 这时微处理机根据指令要 求的固定相及柱温, 从内存中选出所需的温度系数及该固定相换算系数,计算得到在该固定相 及柱温时 $\mathrm{C}_{3}-\mathrm{C}_{9}$ 烃保留指数的计算值; 并自动联机, 按已编排的分析方法操作程序去控制分 析器; 然后进样, 得到色谱流出曲线图, 色谱峰的保留时间及峰面积数据; 再从键盘输入确认色 谱峰为何组分时,容许的保留指数误差范围, 及容许的噪声大小后, 就会打印出分析结果的报 告单,报告单上打印有: 色谱峰号、判定此色谱峰可能的组分名称、色谱蜂的保留时间与保留 指数实验值, 此组分保留指数计算值, 组分的含量. 图 1 是用内径 0.23 毫米长 40 米内壁涂 有 OV-101 的 SCOT 柱, 在柱温 $50.0^{\circ} \mathrm{C}$ 、汽化温度 $200^{\circ} \mathrm{C}$ 及氢火焰检测器温度 $200^{\circ} \mathrm{C}$ 时、氮气 为载气,在线分析重整油的实验结果.

这个自编的色谱峰在线定性程序除了可作上述用途外, 还有以下三个功能：在输入规定 范围内的柱温与固定相名称之后, (甲)可打印出 $\mathrm{C}_{5}-\mathrm{C}_{9}$ 碳氢化合物的全部保留指数计算值; (乙)只打印出指定组分的保留指数计算值;(丙)打印出给定保留指数的组分名称; 即起数据袋 的作用. 这使得我们研制具有人工智能气相色谱仪器的工作,又向前迈进了一步.

本文1982年6月16日收到。 


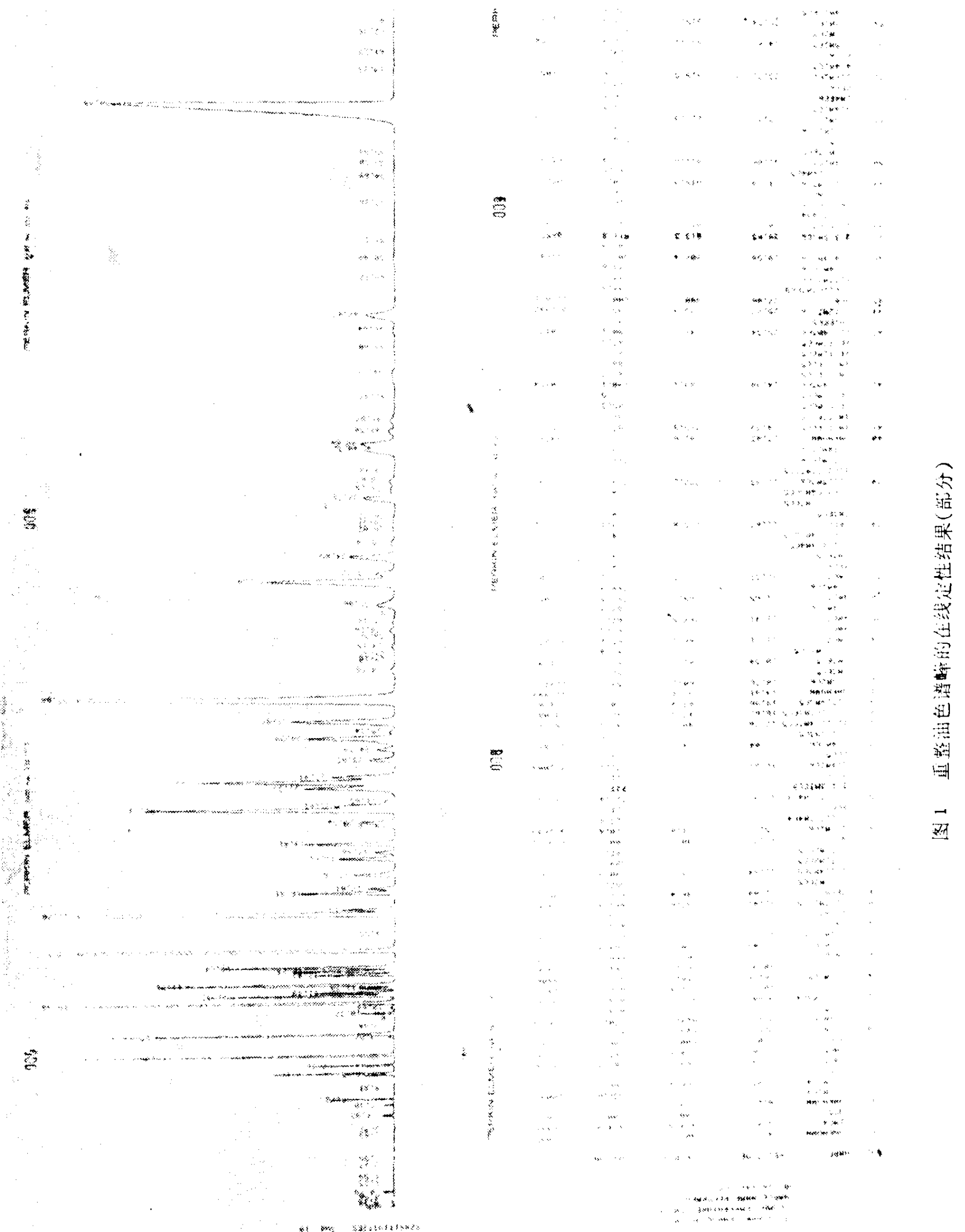

mencos

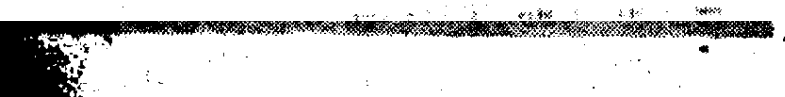




\section{$\Rightarrow$ 考文 触}

[ 1 ] Wham, W. N., Halaby, K. S., Mills, P. M., Eldridge, E. P. \& Thomas, T. G., Amer. Lab., 13(1981), 4: 130.

[2] Perkin-Elmer Co., Anal. Chem., 53(1981), 8: 1012A.

[3] Hewlett Packard Co., Amer. Lab., 13(1981), 8: 39.

[4] Nickel, W., Guidinger, D. C., Brown III, A. C.. Iwao, K. R. \& Marshall, G., ibid., 13(1981), 5: $122,35,36$.

[ 5 ] Rooney, T. A., ibid., 13(1981), 5: 148,83.

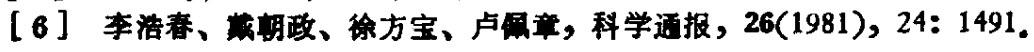

